\title{
Depresión mayor en embarazadas atendidas en el Instituto Nacional Materno Perinatal de Lima, Perú
}

\author{
Matilde Lena Luna Matos, ${ }^{1}$ Joel Salinas Piélago ${ }^{2}$ \\ y Antonio Luna Figueroa ${ }^{3}$
}

Forma de citar Luna Matos ML, Salinas Piélago J, Luna Figueroa A. Depresión mayor en embarazadas atendidas en el Instituto Nacional Materno Perinatal de Lima, Perú. Rev Panam Salud Publica. 2009;26(4):310-4.

RESUMEN Objetivos. Determinar la prevalencia de depresión mayor durante el embarazo y establecer las características sociodemográficas y obstétricas de las mujeres embarazadas con depresión mayor atendidas en la consulta externa de un hospital de Lima, Perú.

Métodos. Estudio descriptivo transversal de asociación simple. Se analizaron 222 gestantes de 16 a 42 años con bajo riesgo obstétrico que acudieron a la consulta externa del Departamento de Obstetricia del Instituto Nacional Materno Perinatal de Lima, Perú, entre el 2 de junio y el 28 de noviembre de 2006. A cada embarazada se le aplicó la escala de depresión de Edimburgo y un cuestionario sobre datos sociodemográficos y obstétricos. Se determinó la relación entre las variables de estudio según la presencia de depresión mayor mediante las pruebas de la $\chi^{2}$ y de la Z, según el tipo de variable.

Resultados. De las 222 gestantes estudiadas, $89(40,1 \%)$ presentaron depresión mayor. Las mujeres casadas tendían a deprimirse menos; las que no planificaron su embarazo y que presentaron complicaciones durante su gestación tendieron a deprimirse más que sus pares. No se encontró que la edad, el número de hijos, la edad gestacional, el nivel educacional, los antecedentes de aborto, el consumo de alcohol u otras sustancias, la falta de apoyo familiar y la percepción de tener problemas de pareja, familiares, económicos o personales influyeran en la frecuencia de depresión.

Conclusiones. La prevalencia de depresión mayor en las gestantes estudiadas fue muy elevada. Se debe prestar especial atención a las mujeres que manifiestan tener un embarazo no planificado y las que sufren complicaciones durante su embarazo, a fin de llegar a diagnósticos tempranos y aplicar tratamientos oportunos.

Palabras clave Trastorno depresivo mayor; complicaciones del embarazo; Perú.

La depresión mayor es una enfermedad crónica recurrente caracterizada por el ánimo depresivo, la pérdida de interés y la baja capacidad para disfrutar (1-2).

\footnotetext{
1 Clínica Ricardo Palma, Lima, Perú. La correspondencia se debe dirigir a Matilde L. Luna, Jirón Mar de Plata No. 178, Urbanización COVIMA, La Molina, Lima 12, Perú. Correo electrónico: lenaluna5pe@yahoo.com

2 Hospital Hermilio Valdizán, Lima, Perú.

3 Instituto Nacional Materno Perinatal, Lima, Perú.
}

Las mujeres son más propensas que los varones a presentar depresión mayor en cualquier período de sus vidas en una proporción de 2:1 (3) y esta es una de las principales causas de enfermedad en mujeres de 15 a 44 años en todo el mundo. Cada año, entre $7 \%$ y $13 \%$ de las mujeres experimenta depresión mayor $(4,5)$, especialmente en los años reproductivos; la prevalencia en embarazadas puede ser de $4 \%$ a $30 \%(6,7)$, en depen- dencia del sistema de tamizaje utilizado. En mujeres gestantes de bajos recursos económicos se han informado frecuencias de hasta $50 \%$ (8).

El estudio de la depresión mayor en embarazadas presenta dificultades, ya que su diagnóstico se basa en los mismos criterios usados para el diagnóstico del cuadro depresivo en mujeres no gestantes. Esto hace difícil distinguir los síntomas de depresión mayor de los que se 
presentan normalmente durante el embarazo, por lo que ha sido necesario desarrollar instrumentos específicos que permitan identificar y cuantificar la depresión mayor en las embarazadas.

La escala de depresión postnatal de Edimburgo (EDPE), desarrollada por Cox y colaboradores en $1987(9,10)$ para evaluar la depresión después del parto, ha demostrado su validez tanto en mujeres gestantes como no gestantes (11). La EDPE es uno de los instrumentos más recomendados para el diagnóstico de la depresión debido a que no toma en cuenta los síntomas somáticos que pudieran confundirse con las molestias propias del embarazo. Esta escala posee una buena sensibilidad $(89,5 \%)$ y especificidad $(72,2 \%)$ en el diagnóstico de la depresión mayor (10).

Actualmente se sabe que la depresión mayor durante el embarazo es tan frecuente o más que la observada después del parto. La prevalencia de síntomas depresivos durante la gestación puede variar ampliamente -entre 2\% y $51 \%(6,7$, 12-19)—, mayor en los dos últimos trimestres del embarazo (17, 18); por su parte, Evans y colaboradores encontraron una prevalencia de $9,1 \%$ a las ocho semanas después del parto (12).

La depresión mayor durante el embarazo está asociada con diferentes factores de riesgo y, aunque constituye una causa frecuente de trastornos psiquiátricos y está bien identificada como un problema de salud, no está suficientemente bien diagnosticada y tratada y aún no se conoce con precisión su incidencia real. A esto puede contribuir que con frecuencia no se documentan adecuadamente los episodios depresivos y su intensidad. Esta situación tiene un impacto negativo sobre la salud de la madre y el hijo, provoca una mayor carga social, clínica y económica, e impide diseñar e implementar intervenciones adecuadas para la prevención y el tratamiento oportunos de las complicaciones asociadas (15). El estudio de la depresión mayor durante el embarazo debe aportar datos útiles que permitan poner en práctica programas eficaces de prevención y tratamiento temprano que contribuyan a mejorar la calidad de la vida de las embarazadas.

El objetivo del presente estudio fue determinar la prevalencia de depresión mayor durante el embarazo y establecer las características sociodemográficas y obstétricas de las mujeres embarazadas con depresión mayor atendidas en la consulta externa de un hospital de Lima, Perú.

\section{MATERIALES Y MÉTODOS}

Se realizó un estudio descriptivo transversal de asociación simple a partir de las 10800 embarazadas de 16 a 42 años con bajo riesgo obstétrico que acudieron a la consulta externa del Departamento de Obstetricia del Instituto Nacional Materno Perinatal (INMP) de Lima, Perú, entre el 2 de junio y el 28 de noviembre de 2006.

Mediante un muestreo probabilístico en una sola etapa se seleccionó una de cada 47 pacientes, a partir de la paciente número 36 , hasta completar una muestra de 227 gestantes, que garantizaba una precisión de $6,5 \%$ y un nivel de confianza de $95 \%$.

A cada embarazada se le aplicó la EDPE con un cuestionario adicional que recababa datos sociodemográficos y obstétricos. Las variables independientes del estudio fueron la edad, el número de hijos, la edad gestacional, el nivel educacional (años de educación formal), el estado civil, si el embarazo fue planificado, los antecedentes de aborto, las enfermedades no obstétricas que presentaba al momento de la aplicación de la encuesta, el consumo de alcohol $\mathrm{u}$ otras sustancias psicoactivas durante el embarazo, la mala relación de pareja y la percepción de algún problema personal, familiar o económico que le produjera estrés al momento de la aplicación de la encuesta. La variable dependiente fue la presencia de depresión mayor durante la gestación (puntuación >13 en la EDPE).

Tres encuestadores psicólogos capacitados para este estudio aplicaron la encuesta en los consultorios del INMP. El número de entrevistas a realizar en un día se determinó a partir de la información de las pacientes atendidas el día anterior hasta completar la muestra establecida. Se excluyeron dos mujeres que se encontraban fuera del grupo de edad preestablecido, una que presentó dificultades para responder los instrumentos aplicados y dos que presentaban alto riesgo obstétrico, por lo que el análisis se realizó a partir de los datos de 222 gestantes.

Las respuestas a cada pregunta de la encuesta se introdujeron en una base de datos y se procesaron con el programa estadístico SPSS versión 13.0. Se calcularon las medidas de tendencia central y de dispersión de las variables cuantitativas y las frecuencias absolutas y relativas de las variables cualitativas. Para deter- minar el grado de relación entre las variables de estudio según la presencia de depresión mayor se utilizaron la prueba de la $\chi^{2}$ y su intervalo de confianza de 95\% (IC95\%). Se utilizó la prueba de la Z para comparar proporciones. El nivel de significación fue de $P \leq 0,05$.

Todas las participantes dieron su consentimiento informado previo. Esta investigación recibió la aprobación del Comité de Ética del INMP, Lima, Perú.

\section{RESULTADOS}

La edad promedio de las participantes fue de 26,9 \pm 6,1 años (mínima: 16; máxima: 42); cerca de $80 \%$ de ellas tenía entre 20 y 34 años. El número promedio de hijos por mujer fue de 1,6 y la puntuación promedio general obtenida en la EDPE fue de 11,4 \pm 4,6 puntos (mínimo: 0; máximo: 24). El tiempo gestacional promedio de las mujeres entrevistadas fue de 28,3 semanas (cuadro 1). Dieciséis $(7,2 \%)$ mujeres se encontraban en el primer trimestre de embarazo, $79(35,6 \%)$ en el segundo y $127(57,2 \%)$ en el tercero.

El nivel educacional predominante fue de secundaria $(61,3 \%)$, seguido de la educación universitaria $(18,0 \%)$; solo $8(3,6 \%)$ tenían educación primaria. En cuanto al estado civil, predominaron las que vivían con sus parejas: 134 $(60,4 \%)$ eran convivientes (unión consensual) y $55(24,8 \%)$ estaban casadas (cuadro 1).

En 125 (56,3\%) casos, el embarazo no fue planificado; $72(32,4 \%)$ mujeres tenían antecedentes de aborto y $96(43,2 \%)$ presentaron complicaciones durante el embarazo actual. De las gestantes, 24 $(10,8 \%)$ refirieron tener una mala relación con su pareja y $11(5,0 \%)$ no contaban con el apoyo de su familia. La gran mayoría $210(94,6 \%)$ de las participantes refirió tener problemas personales, familiares o económicos que le causaban estrés y 5 (2,3\%) consumió alcohol u otras drogas durante el embarazo. Respecto a los problemas de salud durante el embarazo, $21(9,5 \%)$ gestantes refirieron alguna enfermedad como anemia, infección urinaria, hipertensión arterial y epilepsia (cuadro 2).

\section{Depresión mayor}

De las 222 gestantes, $89(40,1 \%)$ tuvieron una puntuación mayor de 13 puntos en la EDPE, indicadora de depresión mayor; las restantes $133(59,9 \%)$ no pre- 
CUADRO 1. Características sociodemográficas de las gestantes estudiadas, según el diagnóstico de depresión mayor, Instituto Nacional Materno Perinatal de Lima, Perú, junio-noviembre de $2006^{a}$

\begin{tabular}{|c|c|c|c|c|c|c|c|}
\hline \multirow[b]{2}{*}{ Característica } & \multicolumn{2}{|c|}{$\begin{array}{c}\text { Total } \\
(n=222)\end{array}$} & \multicolumn{2}{|c|}{$\begin{array}{c}\text { Deprimidas } \\
(n=89)\end{array}$} & \multicolumn{2}{|c|}{$\begin{array}{c}\text { No deprimidas } \\
\quad(n=133)\end{array}$} & \multirow[b]{2}{*}{$P$} \\
\hline & No. & $\%$ & No. & $\%$ & No. & $\%$ & \\
\hline \multicolumn{8}{|l|}{ Estado civil } \\
\hline Soltera & 27 & 12,1 & 13 & 14,6 & 14 & 10,5 & $0,508^{b}$ \\
\hline Conviviente & 134 & 60,4 & 58 & 65,2 & 76 & 57,1 & $0,668^{b}$ \\
\hline Casada & 55 & 24,8 & 13 & 14,6 & 42 & 31,6 & $0,001^{\mathrm{b}}$ \\
\hline Separada & 6 & 2,7 & 5 & 5,6 & 1 & 0,8 & $0,062^{b}$ \\
\hline \multicolumn{8}{|l|}{ Nivel educacional } \\
\hline Primaria & 8 & 3,6 & 4 & 4,5 & 4 & 3,0 & $0,66^{\mathrm{b}}$ \\
\hline Secundaria & 136 & 61,3 & 58 & 65,2 & 78 & 58,6 & $0,85^{b}$ \\
\hline Técnica superior & 38 & 17,1 & 14 & 15,7 & 24 & 18,1 & $0,44^{b}$ \\
\hline Universitaria & 40 & 18,0 & 13 & 14,6 & 27 & 20,3 & $0,15^{b}$ \\
\hline Edad (media $\pm \mathrm{DE}^{\mathrm{c}}$ ) & \multicolumn{2}{|c|}{$26,9 \pm 6,1$} & \multicolumn{2}{|c|}{$26,6 \pm 6,6$} & \multicolumn{2}{|c|}{$27,1 \pm 5,8$} & $0,539^{d}$ \\
\hline Edad gestacional (media $\pm \mathrm{DE}$ ) & \multicolumn{2}{|c|}{$28,3 \pm 9,4$} & \multicolumn{2}{|c|}{$28,1 \pm 9,4$} & \multicolumn{2}{|c|}{$28,3 \pm 9,3$} & $0,841^{d}$ \\
\hline Número de hijos (media $\pm \mathrm{DE}$ ) & \multicolumn{2}{|c|}{$1,6 \pm 1,3$} & \multicolumn{2}{|c|}{$1,5 \pm 1,0$} & \multicolumn{2}{|c|}{$1,7 \pm 1,5$} & $0,191^{d}$ \\
\hline
\end{tabular}

${ }^{a}$ El puntaje promedio en la escala de depresión postnatal de Edimburgo fue de 11,4 4,6 (mínimo: 0; máximo 24).

${ }^{b}$ Prueba de comparación de proporciones de $Z$. Nivel de significación $P<0,05$.

${ }^{\mathrm{C} D E}$ : desviación estándar.

${ }^{d}$ Prueba de la $t$ de Student. Nivel de significación $P \leq 0,05$

CUADRO 2. Características sociales y obstétricas de las gestantes estudiadas, según el diagnóstico de depresión mayor, Instituto Materno Perinatal de Lima, junio-noviembre de 2006

\begin{tabular}{|c|c|c|c|c|c|c|c|c|}
\hline \multirow[b]{2}{*}{ Característica } & \multicolumn{2}{|c|}{$\begin{array}{c}\text { Total } \\
(n=222)\end{array}$} & \multicolumn{2}{|c|}{$\begin{array}{l}\text { Deprimidas } \\
(n=89)\end{array}$} & \multicolumn{2}{|c|}{$\begin{array}{c}\text { No deprimidas } \\
\quad(n=133)\end{array}$} & \multirow[b]{2}{*}{$\chi^{2}$} & \multirow[b]{2}{*}{$P^{a}$} \\
\hline & No. & $\%$ & No. & $\%$ & No. & $\%$ & & \\
\hline \multicolumn{9}{|c|}{ Relación de pareja } \\
\hline Buena & 198 & 89,2 & 75 & 84,3 & 123 & 92,4 & \multirow{2}{*}{3,73} & \multirow{2}{*}{0,053} \\
\hline Mala & 24 & 10,8 & 14 & 15,7 & 10 & 7,6 & & \\
\hline \multicolumn{9}{|l|}{ Apoyo familiar } \\
\hline Sí & 211 & 95,0 & 83 & 93,3 & 128 & 96,2 & \multirow{2}{*}{1,01} & \multirow{2}{*}{0,315} \\
\hline No & 11 & 5,0 & 6 & 6,7 & 5 & 3,8 & & \\
\hline \multicolumn{9}{|c|}{ Embarazo planificado } \\
\hline Sí & 97 & 43,7 & 31 & 34,8 & 66 & 49,6 & \multirow[b]{2}{*}{4,74} & \multirow{2}{*}{0,029} \\
\hline No & 125 & 56,3 & 58 & 65,2 & 67 & 50,4 & & \\
\hline \multicolumn{9}{|c|}{ Antecedente de aborto } \\
\hline Sí & 72 & 32,4 & 32 & 36,0 & 40 & 30,1 & \multirow{2}{*}{0,84} & \multirow{2}{*}{$0,35 \varsigma$} \\
\hline No & 150 & 67,6 & 57 & 64,0 & 93 & 69,9 & & \\
\hline \multicolumn{9}{|c|}{$\begin{array}{l}\text { Complicación durante } \\
\text { el embarazo }\end{array}$} \\
\hline Sí & 96 & 43,2 & 46 & 51,7 & 50 & 37,6 & \multirow{2}{*}{4,07} & \multirow{2}{*}{0,044} \\
\hline No & 126 & 56,8 & 43 & 48,3 & 83 & 62,4 & & \\
\hline \multicolumn{9}{|c|}{$\begin{array}{l}\text { Consumió alcohol u otras } \\
\text { sustancias psicoactivas }\end{array}$} \\
\hline Sí & 5 & 2,3 & 2 & 2,2 & 3 & 2,3 & \multirow{2}{*}{2,3} & \multirow{2}{*}{0,997} \\
\hline No & 217 & 97,7 & 87 & 97,8 & 130 & 97,7 & & \\
\hline \multicolumn{9}{|c|}{$\begin{array}{l}\text { Problema personal, familiar o } \\
\text { económico que cause estrés }\end{array}$} \\
\hline Sí & 210 & 94,6 & 3 & 3,4 & 9 & 6,8 & \multirow{2}{*}{93,1} & \multirow{2}{*}{0,272} \\
\hline No & 12 & 5,4 & 86 & 96,6 & 124 & 93,2 & & \\
\hline \multicolumn{9}{|c|}{$\begin{array}{l}\text { Problemas de salud } \\
\text { durante el embarazo }\end{array}$} \\
\hline Sí & 21 & 9,5 & 13 & 14,5 & 8 & 5,7 & \multirow{2}{*}{4,55} & \multirow{2}{*}{0,033} \\
\hline No & 201 & 90,5 & 76 & 85,5 & 125 & 94,3 & & \\
\hline
\end{tabular}

a Prueba de la $\chi^{2}$. Nivel de significación $P<0,05$.

${ }^{\mathrm{b}}$ Anemia, infección urinaria, hipertensión arterial y epilepsia.

sentaron síntomas de depresión. No se encontraron diferencias significativas entre ambos grupos en cuanto a la edad materna $(P=0,539)$, la edad gestacio- nal $(P=0,841)$ y el número de hijos $(P=0,191)$ (cuadro 1).

En las mujeres gestantes casadas se observó una proporción significativa- mente menor de deprimidas $(P=0,001)$; no se encontró asociación entre la presencia de síntomas depresivos según el nivel educacional $(P>0,05)$ (cuadro 1$)$.

Según los resultados de la EDPE, no se encontraron diferencias respecto a la frecuencia de depresión según la percepción de tener problemas con la pareja $(P=0,053)$, el apoyo familiar $(P=0,315)$ y los antecedentes de aborto $(P=0,359)$. Hubo una proporción significativamente mayor de casos de depresión entre las mujeres que no habían planificado su embarazo que en las que lo habían planificado $(P=0,029)$ y en las que habían tenido complicaciones durante su embarazo actual con respecto a las que no presentaron complicaciones $(P=0,044)$ y en las que presentaron algún problema de salud durante el embarazo con respecto a sus pares que no los presentaron $(P=0,033)$ (cuadro 2).

Finalmente, no se encontraron diferencias estadísticamente significativas con respecto a la frecuencia de casos de depresión entre las mujeres que habían consumido alcohol $\mathrm{u}$ otras sustancias psicoactivas y las que no declararon ese consumo $(P=0,997)$ y entre las mujeres que percibieron tener algún problema económico, familiar o personal que le produjera estrés durante el embarazo $\mathrm{y}$ las que no lo percibieron $(P=0,272)$ (cuadro 2).

\section{DISCUSIÓN}

En el presente estudio se encontró que $40,1 \%$ de las gestantes que acudieron al INMP de Lima sufrían depresión mayor. En un estudio realizado en Perú en 2004 se encontró una prevalencia de depresión mayor de 13,5\% en mujeres con 32 semanas de gestación que vivían en la comunidad (15), mientras en otro realizado en una sala de maternidad de Perú, esta proporción fue de 17,0\% (16). Estos porcentajes son mayores que los informados para la depresión postparto, que van de $8,1 \%$ a $9,1 \%(17-21)$.

En este estudio no se encontraron diferencias significativas entre las gestantes deprimidas y no deprimidas con respecto a algunas características sociodemográficas y obstétricas que se han asociado consistentemente con la depresión. Investigaciones previas han demostrado que los antecedentes de abuso infantil, los trastornos psiquiátricos previos (depresión, ansiedad, abuso de alcohol o sustancias psicoactivas), la alta paridad 
(11), el insuficiente apoyo social, los conflictos de pareja, el embarazo no planificado $(22,23)$, la condición de madre adolescente, primípara o soltera, el bajo nivel educacional (14) y el haber tenido algún aborto previo (24) se asociaron significativamente con las enfermedades psiquiátricas durante el embarazo. En un estudio realizado en mujeres de 14 a 37 semanas de gestación evaluadas con la EDPE en Indonesia se encontró una elevada prevalencia de depresión asociada con la dependencia de sustancias psicoactivas y los problemas familiares y de pareja (25).

Tal como se podría esperar, se encontró que las mujeres casadas tendían a deprimirse menos y que las gestantes que no planificaron su embarazo o que pre- sentaron complicaciones o problemas de salud durante su embarazo tendieron a deprimirse más que sus pares. Sin embargo, no se encontró que la edad, el número de hijos, la edad gestacional, el nivel educacional, los antecedentes de aborto, el consumo de alcohol $\mathrm{u}$ otras sustancias psicoactivas, la falta de apoyo familiar y la percepción de tener problemas de pareja, familiares, económicos o personales influyeran en la frecuencia de depresión.

Estos resultados confirman la importancia de entender el contexto social, cultural y económico en que se desarrolla el embarazo -incluso desde antes de la concepción - para interpretar adecuadamente el posible origen de la depresión mayor de las mujeres gestantes (25). Se debe tener presente que la depresión mayor aumenta el riesgo de enfermedades físicas, complicaciones obstétricas, problemas sociales y conductuales, así como de la depresión puerperal, con un efecto negativo en la embarazada, el sistema de salud y la familia de las gestantes $(17,18)$.

En conclusión, la prevalencia de depresión mayor en las gestantes estudiadas fue muy elevada. Se debe prestar especial atención a las mujeres que manifiestan tener un embarazo no planificado y las que sufren complicaciones o problemas de salud durante su embarazo, a fin de llegar a diagnósticos tempranos y aplicar tratamientos oportunos. Se recomienda observar a las gestantes que refieren tener mala relación con su pareja.

\section{REFERENCIAS}

1. American Psychiatric Association. Diagnostic and statistical manual of mental disorders. 4th ed. Washington, D.C.: American Psychiatric Association; 1994.

2. World Health Organization. The ICD-10 classification of mental and behavioral disorders. Geneva: WHO; 1992.

3. Picech R, Flores L, Lanser R, Cremonte M. Depresión y embarazo. Rev Post-Grado Med (Corrientes, Argentina). 2005;145:5-8.

4. Australian Institute of Health and Welfare. National health priority areas report: mental health 1998. Canberra: Commonwealth Department of Health and Aged Care, AIHW; 1999.

5. Berardi D, Leggieri G, Ceroni GB, Rucci P, Pezzoli A, Paltrinieri E, et al. Depression in primary care: a nationwide epidemiological survey. Fam Pract. 2002;19:397-400.

6 Johanson R, Chapman G, Murray D, Johnson I, Cox J. The North Staffordshire Maternity Hospital prospective study of pregnancyassociated depression. J Psychosom Obstet Gynaecol. 2000;21:93-7.

7. McKee M, Cunningham M, Jankowksi K, Zayas L. Health-related functional status in pregnancy: relationship to depression and social support in a multi-ethnic population. Obstet Gynecol. 2001;97:988-93.

8. Chokka P. Postpartum depression. Part 1. Can J Contin Med Educ. 2002;14(4):37-45.

9. Cox JL, Holden JM, Sagovsky R. Detection of postnatal depression: development of the 10item Edinburgh Postnatal Depression Scale. Br J Psychiatry. 1987;150:782-6.

10. Vega-Dienstmaier J, Mazzotti G, Stucchi-Portocarrero S, Salinas-Piélago J, Gallo C, Poletti
G. Validación de la escala de depresión de Edinburgh en mujeres que no están en el período postparto. Rev Neuropsiquiatr. 1997; 60:283-93.

11. Alviki A, Heyerdahl S, Haldorsen T, Lindermann R. Alcohol use before and during pregnancy: a population-based study. Acta Obstet Gynecol. 2006;85:1292-8.

12. Evans J, Heron J, Francomb H, Oke S, Golding J. Cohort study of depressed mood during pregnancy and alter childbirth. Br Med J. 2001;323;257-60.

13. Bennett HA, Einarson A, Taddio A, Koren G, Einarson TR. Prevalence of depression during pregnancy: systematic review. Obstet Gynecol. 2004;103:698-709.

14. Bowen A, Mujaharine N. Antenatal depression. Can Nurse. 2006;102:27-30.

15. Qiu C, Sánchez S, Lam N, García, Williams M. Association of depression symptoms whith preeclampsia: results from a Peruvian case study. BMC Womens Health. 2007;7:15.

16. Josefsson A, Berg G, Nordin C, Sydsjo G. Prevalence of depressive symptoms in late pregnancy and postpartum. Acta Obstet Gynecol Scand. 2001;80:251-5.

17. Sánchez S, Qiu C, Perales M, Lam N, García $\mathrm{P}$, Williams $\mathrm{M}$. Intimate partner violence and preeclampsia among Peruvian women. Eur J Obstet Gynecol Reprod Biol. 2008;137(1): 50-5.

18. Rodriguez M, Heilemann M, Fielder E, Ang A, Navarez F, Mangione C. Intimate partner violence, depression and PTSD among pregnant women. Ann Fam Med. 2008;6:1.

19. Orr S, Miller C. Maternal depressive symptoms and the risk of poor pregnancy outcome: review of the literature and preliminary findings. Epidemiol Rev. 1995;17:165-71.

20. Ryan D, Milia L, Misri N. Depression during pregnancy. Can Fam Physician. 2005;51: 1087-93.

21. Rubertsson C, Wickberg B, Gustavsson P, Radestad I. Depressive symptoms in early pregnancy, two months and one year postpartum-prevalence and psychosocial risk factors in a national Swedish sample. Arch Women Mental Health. 2005;8:97-104.

22. Varma D, Chandra P, Thomas T, Carey M. Intimate partner violence and sexual coercion among pregnant women in India: relationship with depression and post-traumatic stress disorder. J Affect Disord. 2007;102(1): 227-35.

23. Cripe S, Sánchez S, Perales M, Lam N, García $\mathrm{P}$, Williams A. Association of intimate partner physical and sexual violence with unintended pregnancy among pregnant women in Peru. Int J Gynocol Obstet. 2008;100(2):104-8.

24. Adewuya AO, Ola BA, Aloba OO, Dada AO, Fasoto OO. Prevalence and correlates of depression in late pregnancy among Nigerian women. Depress Anxiety. 2007;24:15-21.

25. Andajani-Sutjahjo S, Manderson L, Astbury J. Complex emotions, complex problems: understanding the experiences of perinatal depression among new mothers in urban Indonesia. Cult Med Psychiatry. 2007;31:101-22.

Manuscrito recibido el 10 de enero de 2008. Aceptado para publicación, tras revisión, el 30 de octubre de 2008. 
ABSTRACT Objectives. To determine the prevalence of major depression during pregnancy and to define the sociodemographic and obstetric characteristics of pregnant women with major depression treated by the outpatient clinic at a hospital in Lima, Peru.

\section{Major depression in pregnant women served by the National Materno-Perinatal Institute in Lima, Peru}

Key words Depressive disorder, major; pregnancy complications; Peru.
Methods. A descriptive, cross-sectional study with simple association. The analysis consisted of 222 pregnant women 16-42 years of age with low obstetric risk who had visited the outpatient services of the Obstetrics Department at the National MaternoPerinatal Institute in Lima, Peru, from 2 June-28 November 2006. Each pregnant woman was given the Edinburgh Depression Scale and a questionnaire on sociodemographic and obstetric data. The relationship between the study variables was determined by the presence of major depression revealed through either $\chi^{2}$ or $Z$ tests, depending on variable type.

Results. Of the 222 pregnant women studied, 89 (40.1\%) had major depression. Fewer married women tended to be depressed; those with unplanned pregnancies and pregnancy complications were more often depressed than their peers. Age, number of children, gestational stage, educational level, history of abortion/miscarriage, consumption of alcohol or other drugs, lack of family support, and perceived marital, family, or financial problems were not found to influence depression frequency.

Conclusions. The prevalence of major depression among pregnant women was very high. Special attention should be given to women with unplanned pregnancies and those experiencing complications during pregnancy, so that an early diagnosis can be made and appropriate treatment offered.

\section{Atención comunitaria a personas con trastornos psicóticos}

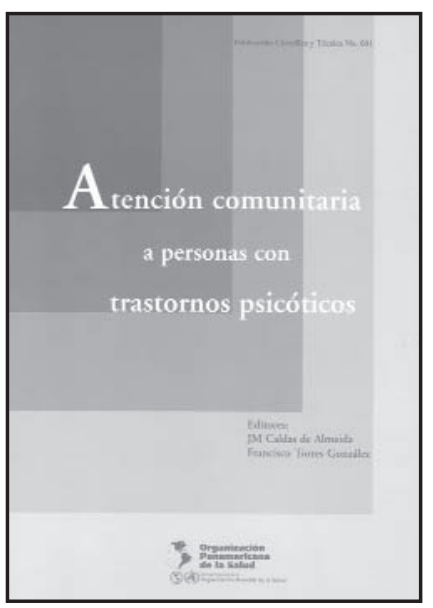

$2005,152 \mathrm{pp}$.

ISBN 9275316015

Código: PC 601

Precio: US\$18,00 en América

Latina y el Caribe/ US $\$ 25,00$ en el resto del mundo
En este libro se presentan 13 guías operativas para desarrollar programas comunitarios de atención a personas con trastornos psicóticos que se puedan integrar a los planes nacionales de salud mental. La primera parte de esta obra presenta las bases epidemiológicas y científicas de los trastornos mentales, así como las estrategias utilizadas en la formulación de programas de atención comunitaria para quienes los padecen. La segunda parte la constituyen las guías operativas, en las que se recomiendan medidas para desarrollar mejores programas de atención comunitaria en el marco de la salud mental.

Esta publicación será de gran utilidad para los responsables de formular políticas de salud mental, médicos encargados de las unidades de salud mental en las diversas instituciones de atención comunitaria de salud, psiquiatras, psicólogos, personal de enfermería, proveedores de atención comunitaria, estudiantes de psicología, psiquiatría y salud pública, así como para lectores interesados en la salud mental y la atención comunitaria. 